\title{
ReaR
}

\section{Impacto de las terapias de depuración extrarrenal en la concentraciones plasmáticas de los antimicrobianos en $\mathrm{UCl}$.}

Domingo Chiva E, Charco Roca LM.

Complejo Hospitalario Universitario de Albacete.

\section{Resumen}

En los pacientes críticos con insuficiencia renal aguda es esencial manejar cuidadosamente la dosis de los antimicrobianos, especialmente si se requieren terapias de reemplazo renal continuas. La insuficiencia renal aguda conlleva por sí misma modificaciones clínicamente significativas de los parámetros farmacocinéticos de los fármacos, y la necesidad de terapias de reemplazo renal representa una variable adicional que debe considerarse para evitar una terapia antimicrobiana inadecuada. En muchos casos la eliminación extracorpórea de antimicrobianos puede ser relevante con el consiguiente riesgo de fracaso terapéutico relacionado con la infradosificación y/o posible aparición de resistencias bacterianas.

En este escenario clínico, el conocimiento de las propiedades farmacocinéticas y farmacodinámicas de los distintos antimicrobianos es esencial para adaptar las dosis y/o intervalos de dosificación.

El propósito de esta revisión es mostrar un resumen actualizado de la evidencia actual de este fenómeno y orientar al posible ajuste de fármacos.

Para la elaboración de este manuscrito se ha llevado a cabo una revisión no sistemática de artículos indexados en MEDLINE (PubMed) de los últimos quince años, fecha de actualización diciembre 2019, utilizando las palabras clave "critically ill", "acute kidney injury", "antimicrobials", "continuous renal replacement therapy" y "extracorporeal clearance". Se excluyeron aquellos artículos realizados en población pediátrica y/o que no estuvieran publicados en inglés o español.

\section{Introducción}

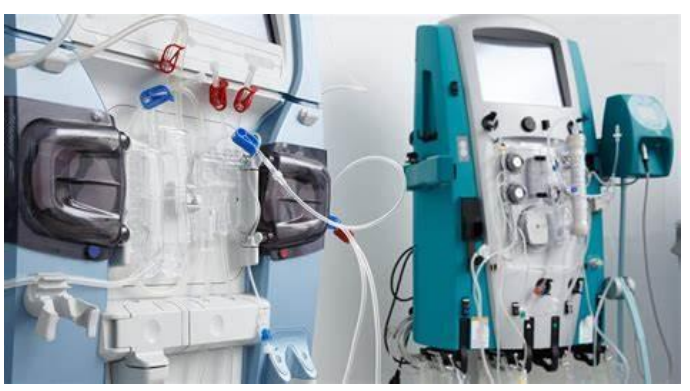

En los pacientes críticos con insuficiencia renal aguda es esencial manejar cuidadosamente la dosis de los antimicrobianos, especialmente si se requieren terapias de reemplazo renal continuas. La insuficiencia renal aguda conlleva por sí misma modificaciones clínicamente significativas de los parámetros farmacocinéticos de los fármacos, y la necesidad de terapias de reemplazo renal representa una variable adicional que debe considerarse para evitar una terapia antimicrobiana inadecuada. En muchos casos la eliminación extracorpórea de antimicrobianos puede ser relevante con el consiguiente riesgo de fracaso terapéutico relacionado con la infradosificación y/o posible aparición de resistencias bacterianas.

En este escenario clínico, el conocimiento de las propiedades farmacocinéticas y farmacodinámicas de los distintos antimicrobianos es esencial para adaptar las dosis $\mathrm{y} / \mathrm{o}$ intervalos de dosificación.

El propósito de esta revisión es mostrar un resumen actualizado de la evidencia 
actual de este fenómeno y orientar al posible ajuste de fármacos.

Para la elaboración de este manuscrito se ha llevado a cabo una revisión no sistemática de artículos indexados en MEDLINE (PubMed) de los últimos quince años, fecha de actualización diciembre 2019, utilizando las palabras clave "critically ill", "acute kidney injury", "antimicrobials", "continuous renal replacement therapy" y "extracorporeal clearance". Se excluyeron aquellos artículos realizados en población pediátrica $\mathrm{y} / \mathrm{o}$ que no estuvieran publicados en inglés $\mathrm{O}$ español.

La disfunción renal aguda es una complicación grave y frecuente en el ámbito de la Unidad de Cuidados Intensivos (UCI) que se asocia al empleo de terapias continuas de reemplazo renal (TRRC)(1). Pero el uso de estas técnicas no está exento de complicaciones, como las bacteriemias, coagulación del circuito, la disfunción del catéter, las lesiones vasculares y el riesgo de sangrado $(2,3)$, entre otras. Dentro de estas complicaciones o efectos secundarios debemos incluir las alteraciones en las propiedades farmacocinéticas de los fármacos que comúnmente se administran en el contexto del paciente crítico, por las cuales es necesario saber predecir su comportamiento en el contexto de la terapia para no incurrir en fracaso terapéutico.

Son muchos los fármacos que pueden verse afectados por las TRRC. En algunos, como los sedantes, anticoagulantes, drogas vasoactivas, etc. estas alternaciones son fácilmente monitorizables, detectables y corregibles; sin embargo, en otros, como los antimicrobianos es más complicado y solo es posible en algunos casos mediante la determinación de concentraciones plasmáticas de estos.
El propósito de esta revisión es mostrar un resumen actualizado de la evidencia actual de este fenómeno y orientar al posible ajuste de fármacos.

Para la elaboración de este manuscrito se ha llevado a cabo una revisión no sistemática de artículos indexados en MEDLINE (PubMed) de los últimos quince años, fecha de actualización diciembre 2019, utilizando las palabras clave "critically ill", "acute kidney injury", "antimicrobials", "continuous renal replacement therapy" $y$ "extracorporeal clearance". Se excluyeron aquellos artículos realizados en población pediátrica $\mathrm{y} / \mathrm{o}$ que no estuvieran publicados en inglés $\mathrm{o}$ español.

\section{Alteraciones farmacocinéticas en pacientes con técnicas de remplazo renal}

El uso de las TRRC supone una alteración relevante en la farmacocinética de muchos antimicrobianos, siendo de una complejidad superior a la que ocurre durante la hemodiálisis intermitente debido al alto número de variables implicadas, incluyendo tanto propiedades propias del fármaco como factores relacionados con el paciente $o$ con la técnica de reemplazo renal utilizada (tabla 1) $(2,4)$.

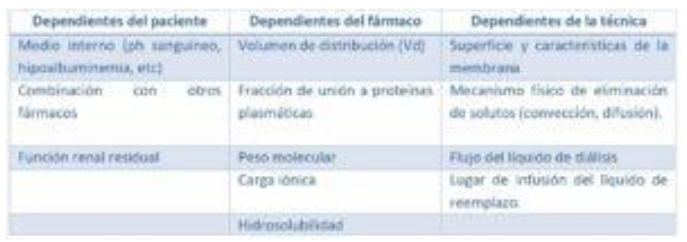

Tabla 1. Determinantes de la eliminación de fármacos mediante $\operatorname{TRRC}(4,9)$.

\section{Mecanismos de eliminación de fármacos}

La eliminación de solutos de la sangre a través de membranas semipermeables durante los procesos de remplazo renal 
puede ocurrir por diferentes procesos fisicoquímicos:

- DIFUSIÓN: La difusión representa el principio fundamental de diálisis $\mathrm{y}$ consiste en el transporte pasivo de los solutos a través de una membrana semipermeable debido al gradiente de concentración generado a ambos lados de ésta. Este proceso está regido por la Ley de Fick: la velocidad de difusión de las moléculas por unidad de tiempo (J) es directamente proporcional al coeficiente de difusión (depende de las características de la molécula), la superficie disponible para la difusión, el gradiente de concentración a ambos lados de la membrana e inversamente proporcional al espesor de la membrana. En este proceso influyen tanto la diferencia de concentración como el tamaño de poro de la membrana. Las membranas de alto flujo de diálisis presentan tamaños de poro de membrana más grande que las membranas tradicionales, por lo que ciertos fármacos con bajo peso molecular podrían atravesarlas y depurarse de la circulación sistémica.

- CONVECCIÓN/ULTRAFILT

RACIÓN: Representa la base de la hemofiltración y se basa en el paso de solutos arrastrados por un fluido a través de una membrana debido a la diferencia de presión que éste ejerce en uno de los lados de la membrana. La tasa de ultrafiltración es directamente proporcional a la diferencia de presión transmembrana y al área de membrana disponible. La pérdida de fármacos por hemofiltración es independiente del tamaño de molécula, considerando que la mayoría de estas membranas tiene tamaños de poro superiores al tamaño de la mayor parte de antimicrobianos (30.000-50.000 Da). Además, la reposición del ultrafiltrado generado con fluidos para preservar el volumen supone una dilución de fármaco en el torrente circulatorio, bien pre-filtración (pre-dilución) o tras el filtrado (post-dilución), que afectará a la concentración alcanzada y al aclaramiento del fármaco.

Cómo norma general, la eliminación de fármacos por sistemas de remplazo renal es Hemodiafiltración> Hemofiltración> Hemodiálisis. El aclaramiento a través de estas técnicas puede variar en gran medida en función del flujo empleado y las propiedades fisicoquímicas del fármaco (5).

\section{Factores que afectan a la eliminación de antimicrobianos}

\section{Propiedades del fármaco}

Las terapias de reemplazo renal sustituyen la función del riñón por lo que es de esperar que los fármacos que más se van a ver afectados con su utilización son aquellos con un aclaramiento principalmente renal.

De manera general, los fármacos hidrofílicos son fármacos con bajo volumen de distribución $(\mathrm{Vd})$ que permanecen en el torrente circulatorio y son eliminados predominantemente por vía renal. Los antibióticos que cumplen estas características son: betalactámicos, glicopéptidos y aminoglucósidos. Como excepciones podemos destacar la de ceftriaxona, que se elimina por excreción biliar a pesar de ser hidrófilo, y la de las quinolonas como levofloxacino y ciprofloxacino que 
aunque son hidrófobos, se eliminan por excreción renal (6). Por el contrario, los fármacos lipofílicos presentan un alto $\mathrm{Vd}$, y se concentran en su mayor parte en tejidos periféricos, viéndose, por tanto, su eliminación menos afectada por alteraciones a nivel renal. En general, a mayor $\mathrm{Vd}$, el fármaco se verá menos afectados por variaciones en la eliminación renal.

Por todo esto, los fármacos hidrofílicos requerirán dosis más elevadas en pacientes con TRRC en comparación con pacientes anúricos, mientras que los fármacos de carácter lipófilo no requerirán esta suplementación de dosis, pues el porcentaje de pérdida respecto al acumulado en tejidos será mínimo. En la tabla $2(4,5,7,8)$ se muestran las principales características

farmacocinéticas de los antibióticos más comúnmente utilizados.

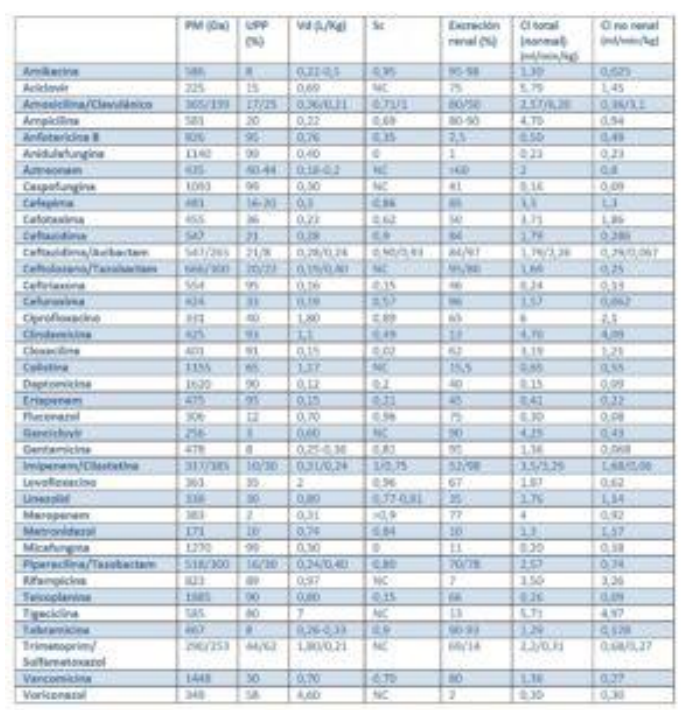

Tabla 2. Propiedades farmacocinéticas de los antimicrobianos $(4,5,7,8)$. Abreviaturas: $\mathrm{Cl}$ total $=$ Aclaramiento total; $\mathrm{Cl}$ no renal = Aclaramiento no renal; $\mathrm{NC}=$ No conocido; $\mathrm{PM}$ $=$ Peso molecular; $\mathrm{Sc}=$ Coeficiente de cribado o Sieving; $\mathrm{Vd}=$ Volumen de distribución.

Por otro lado, considerando que el tamaño de poro no filtra proteínas plasmáticas $(>50,000 \mathrm{Da})$, únicamente el fármaco libre (no unido a proteínas) será eliminado por el procedimiento de remplazo renal. Es por ello que a mayor unión a proteínas plasmáticas (UPP), menor será su eliminación por el sistema. Este concepto puede cuantificarse mediante el llamado Coeficiente de Cribado o Sieving (Cs), que refleja el ratio entre la concentración de fármaco en ultrafiltrado y plasma.

$$
C s=C_{u f} / C p
$$

Por último, debemos de considerar que hay factores que pueden afectar a este coeficiente. Así, en el paciente crítico es frecuente la hipoalbuminemia. En esta situación la cantidad de fármaco libre puede incrementarse de forma considerable, favoreciendo su eliminación. Además, la acumulación de ciertos solutos en pacientes con insuficiencia renal grave puede alterar el grado de unión del fármaco a las proteínas plasmáticas, contribuyendo a mantener una mayor cantidad de fármaco libre.

Por otro lado, parte del fármaco puede adherirse a las membranas del hemofiltro y perderse de la circulación, si bien este efecto solo tendría repercusión durante las fases iniciales del nuevo filtro, ya que dicha adhesión es saturable e impide que se pierda más fármaco.

\section{Propiedades del procedimiento de remplazo renal}

Respecto al equipo de remplazo renal, la eliminación del fármaco dependerá de la superficie del filtro, del flujo de hemofiltración, del modo de remplazo del fluido y del uso de técnicas combinadas de dilución/convención o exclusivamente una de ellas. 


\section{Ajuste de dosis de antimicrobianos durante TRRC}

Con el objetivo de maximizar la eficacia y reducir la toxicidad, la dosificación de los antimicrobianos debe enfocarse para alcanzar el objetivo PK/PD más apropiado.

La fracción de aclaramiento determina si el tratamiento de depuración extracorpórea puede o no implicar un cambio en la dosificación de un determinado fármaco. Si la molécula se elimina por TRRC pero su ruta principal de eliminación es el hígado, la aportación del TRRC puede no llegar al $25 \%$ del aclaramiento total y, por tanto, no tener trascendencia en la dosis. Sólo cuando el aclaramiento extracorpóreo implica más del 25\% del aclaramiento normal del fármaco su fracción de aclaramiento será considerada como significativa e implicará cambios en la dosificación $(9,10)$.

Para calcular la dosis a utilizar en TRRC $(5,9,10) \quad$ en aquellos antimicrobianos en los que es posible determinar los niveles plasmáticos se deberá utilizar la siguiente fórmula:

$$
D_{T R R C}=\left(C_{\text {objetivo }}-C_{\text {actual }}\right) \times V_{d} \times \mathrm{kg}
$$

Sin embargo si no es posible la determinación de niveles plasmáticos del antimicrobiano, la forma de conocer que aclaramiento sufre el fármaco por la influencia de la TRRC será:

- Si la reposición de fluidos se realiza postdilución:

\section{$C l_{H F}=Q_{U F} \times C s$}

- Si la reposición de fluidos se hace antes de la filtración, considerando que el plasma se ha diluido previamente a la entrada en el hemofiltro, el aclaramiento dependerá también del factor de dilución:

$$
C l_{H F}=Q_{U F} \times C s \times\left(Q_{B F} / Q_{B F}+Q_{R F}\right)
$$

En base a estos datos, es posible estimar la dosis necesaria para un paciente, mediante la dosis habitual y el porcentaje de fármaco que es aclarado:

$$
D_{\text {necesaria }}=D_{\text {habitual }} \times\left[\left(C l_{\text {renal }}+C l_{H F}+C l_{\text {no renal }}\right) / C l_{\text {total }}\right]
$$

Conociendo el aclaramiento total y el no renal de los fármacos basándonos en la literatura (tabla 2), podemos calcular la dosis requerida.

Suponiendo que no existe aclaramiento renal durante la TRRC, la ecuación se simplifica:

$$
D_{\text {necesaria }}=D_{\text {habitual }} \times\left(C l_{H F}+C l_{\text {no renal }}\right) / C l_{\text {total }}
$$

Cabe destacar que existen importantes diferencias entre las dosis calculadas bajo este modelo teórico y las dosis recomendadas por las guías de práctica clínica. Las guías de tratamiento basan sus recomendaciones en estudios publicados, habitualmente sobre un grupo reducido de pacientes, en muchas ocasiones fuera del entorno de las UCI. Esta elevada variabilidad de concentraciones observada obedece en gran parte a cambios en el paciente con fracaso renal (incluyendo peso, proteínas plasmáticas y aclaramiento no renal de fármacos) como en el equipo utilizado (sistema de hemofiltración, flujo o membrana utilizada por el sistema).

A pesar de que estas fórmulas no han sido validadas en la práctica clínica, la idea de adecuar la dosis de 
antimicrobiano entendiendo su eliminación puede ser una alternativa para reflexionar (5).

No obstante, estas fórmulas presentan importantes limitaciones, como el no considerar el empleo de diferentes formas de dosificación de los antimicrobianos, tales como perfusiones extendida o continúas de betalactámicos, para conseguir los objetivos $\mathrm{pk} / \mathrm{pD}$.

Por último, cabe señalar que la severidad del paciente y la sensibilidad de la cepa responsable de la infección también tienen que tenerse en cuenta a la hora de dosificar los antimicrobianos en este grupo de pacientes (5).

\section{Conclusiones y recomendaciones}

En los pacientes críticos, la insuficiencia renal aguda puede dar lugar a modificaciones clínicamente significativas de los parámetros farmacocinéticos antibacterianos. De manera general, en aquellos fármacos cuya excreción sea predominantemente renal, las TRRC supondrán una eliminación significativa $y$ por lo general, se requiere un ajuste de la dosis del fármaco si el aclaramiento fisiológico del fármaco es superior al $25 \%$.

Los parámetros farmacocinéticos más importantes, útiles para determinar la importancia de la eliminación extracorpórea de un fármaco, son: peso molecular (PM), UPP y Vd. Sin embargo, para fármacos con volumen de distribución elevado, la eliminación del fármaco mediante las TRRC puede ser insignificante a pesar de tener PM bajo y/o UPP baja.

Finalmente, cabe destacar que cualquier intento de simplificar el ajuste de dosis de antimicrobianos en la práctica clínica puede verse desafiado por la alta variabilidad intra e interindividual de los pacientes críticos. Por lo tanto, para los antimicrobianos con rango terapéutico estrecho, la monitorización farmacocinética mediante niveles plasmáticos es esencial y se recomienda siempre para guiar el ajuste de las dosis del fármaco en un entorno tan multifactorial y rápidamente variable, como es la UCI.

\section{Listado de abreviaturas:}

Cs: Coeficiente de cribado o Sieving.

$\mathrm{C}_{\text {objetivo: }}$ Concentración plasmática objetivo.

$\mathrm{C}_{\text {actual: }}$ Concentración plasmática actual.

Cl total: Aclaramiento total.

$\mathrm{Cl}$ no renal: Aclaramiento no renal.

$\mathrm{Cl}$ renal: Aclaramiento renal.

$\mathrm{Cl}_{\mathrm{HF}}$ : Aclaramiento del fármaco mediante hemofiltración.

Cp: Concentración plasmática.

$\mathrm{C}_{\mathrm{uf}}$ : Concentración de fármaco en ultrafiltrado.

Da: Daltons.

DTRRC: Dosis para TRRC.

PM: Peso molecular.

$\mathrm{QBF}_{\mathrm{BF}}: \mathrm{F}$ lujo de sangre.

$\mathrm{Q}_{\mathrm{RF}}$ : Flujo de líquido de reemplazo.

QuF: Flujo de hemofiltración.

TRRC: Terapias continúas de reemplazo renal.

Vd: Volumen de distribución. 
UCI: Unidad de cuidados intensivos.

UPP: Unión a proteínas plasmáticas.

\section{Bibliografía}

1. Manejo actual de las terapias continúas de reemplazo renal: Estudio epidemiológico multicéntrico. Med Intensiva. 2017;41(4):216-22. (HTML)

2. John S, Eckardt KU. Renal replacement strategies in the ICU. Chest 2007;132:1379-88. (HTML)

3. Ricci Z, Romagnoli S.Technical Complications of Continuous Renal Replacement Therapy. Contrib Nephrol. 2018;194:99-108. (PubMed)

4. Pistolesi V, Morabito S, Di Mario F, Regolisti G, Cantarelli C, Fiaccadori E. A Guide to Understanding Antimicrobial Drug Dosing in Critically Ill Patients on Renal Replacement Therapy. Antimicrob Agents Chemother. 2019 Jul 25;63(8):e00583-19. (PubMed)

5. Gimeno R, Ruiz-Ramos J. Pacientes con circuitos extracorpóreos en: Tratamiento diferencial en pacientes en situaciones especiales. Curso PROA para médicos intensivistas. Sociedad Española de Medicina Intensiva, Crítica y Unidades Coronarias (SEMICYUC). Grupo de trabajo de enfermedades infecciosas y sepsis (GTEIS) y Sociedad Española de Farmacia Hospitalaria (SEFH). Mayo 2019.
6. Pea F, Viale P, Pavan F, Furlanut M. Pharmacokinetic considerations for antimicrobial therapy in patients receiving renal replacement therapy. Clin Pharmacokinet 2007; 46 (12): 997-1038. (PubMed)

7. Heintz BH, Matzke GR, Dager WE. Antimicrobial dosing concepts and recommendations for critically ill adult patients receiving continuous renal replacement therapy or intermittent hemodialysis. Pharmacotherapy 2009 May;29(5):562-77. (PubMed)

8. Echeverri J, Molano A. Ajuste de fármacos y nutrición en terapias de reemplazo renal continuo. Gac Med Mex. 2018; $154 \quad$ (Supp 1): S48S60. (PubMed)

9. Maynar J, Sánchez-Izquierdo J.A. Dosificación de fármacos durante los tratamientos de depuración extracorpórea de la sangre. NefroPlus 2010;3(3):20-26.

10. Schetz M. Drug dosing in continuous renal replacement therapy: general rules. Curr Opin Crit Care. 2007 Dec;13(6):645-51. (PubMed)

Correspondencia al autor

Esther Domingo Chiva

estherdomingochiva@hotmail.com

Facultativo Especialista de Área. Servicio de Farmacia Hospitalaria.

Complejo Hospitalario Universitario de Albacete.

Aceptado para el blog en enero de 2021 